\title{
Changes in Smoking and Vaping over 18 Months among Smokers and Recent Ex-Smokers: Longitudinal Findings from the 2016 and 2018 ITC Four Country Smoking and Vaping Surveys
}

\author{
Shannon Gravely ${ }^{1}$, Gang Meng ${ }^{1}$, K. Michael Cummings ${ }^{2}{ }^{\circledR}$, Andrew Hyland ${ }^{3}$, Ron Borland ${ }^{4}$, \\ David Hammond ${ }^{5}$, Richard J. O'Connor ${ }^{3}$, Maciej L. Goniewicz ${ }^{3}{ }^{\circledR}$, Karin A. Kasza ${ }^{3}$, \\ Ann McNeill 6,7 (D), Mary E. Thompson ${ }^{8}$, Sara C. Hitchman ${ }^{6}$, David T. Levy ${ }^{9}$, \\ James F. Thrasher ${ }^{10,11}$, Anne C. K. Quah ${ }^{1}$, Nadia Martin ${ }^{1}$, Janine Ouimet ${ }^{1}$, \\ Christian Boudreau ${ }^{8}$ and Geoffrey T. Fong ${ }^{1,5,12, *}$ \\ 1 Department of Psychology, University of Waterloo, Waterloo, ON N2L 3G1, Canada; \\ shannon.gravely@uwaterloo.ca (S.G.); gmeng@uwaterloo.ca (G.M.); ackquah@uwaterloo.ca (A.C.K.Q.); \\ nadia.martin@uwaterloo.ca (N.M.); j2ouimet@uwaterloo.ca (J.O.) \\ 2 Department of Psychiatry \& Behavioral Sciences, Medical University of South Carolina, Charleston, \\ SC 29425, USA; cummingk@musc.edu \\ 3 Department of Health Behavior, Roswell Park Comprehensive Cancer Center, Buffalo, NY 14203, USA; \\ Andrew.Hyland@RoswellPark.org (A.H.); richard.o'connor@roswellpark.org (R.J.O.); \\ Maciej.Goniewicz@RoswellPark.org (M.L.G.); karin.kasza@roswellpark.org (K.A.K.) \\ 4 School of Psychological Sciences, University of Melbourne, Melbourne, VIC 3010, Australia; \\ rborland@unimelb.edu.au \\ 5 School of Public Health and Health Systems, University of Waterloo, Waterloo, ON N2L 3G1, Canada; \\ david.hammond@uwaterloo.ca \\ 6 Addictions Department, Institute of Psychiatry, Psychology \& Neuroscience, King's College London, \\ London WC2R 2LS, UK; ann.mcneill@kcl.ac.uk (A.M.); sara.hitchman@kcl.ac.uk (S.C.H.) \\ 7 Shaping public health policies to reduce inequalities \& harm (SPECTRUM), The University of Edinburgh, \\ Edinburgh, EH8 9YL, UK \\ 8 Department of Statistics and Actuarial Science, University of Waterloo, ON N2L 3G1, Canada; \\ methompson@uwaterloo.ca (M.E.T.); cboudreau@uwaterloo.ca (C.B.) \\ 9 Lombardi Comprehensive Cancer Center, Georgetown University, Washington, DC 20057, USA; \\ dl777@georgetown.edu \\ 10 Department of Health Promotion, Education \& Behavior, Arnold School of Public Health, \\ University of South Carolina, Columbia, SC 29208, USA; THRASHER@mailbox.sc.edu \\ 11 Tobacco Research Department, Center for Population Health Research, National Institute of Public Health, \\ Cuernavaca 62100, Mexico \\ 12 Ontario Institute for Cancer Research, Toronto, ON M5G 0A3, Canada \\ * Correspondence: geoffrey.fong@uwaterloo.ca
}

Received: 13 July 2020; Accepted: 23 September 2020; Published: 27 September 2020

Abstract: This descriptive study of smokers (smoked at least monthly) and recent ex-smokers (quit for $\leq 2$ years) examined transitions over an 18 month period in their smoking and vaping behaviors. Data are from Waves 1 (W1: 2016) and 2 (W2: 2018) of the ITC Four Country Smoking and Vaping Survey, a cohort study of adult $(\geq 18+)$ smokers, concurrent users (smoke and vape), and recent ex-smokers from Australia, Canada, England, and the United States (US). Respondents ( $\mathrm{N}=5016)$ were classified according to their smoking and vaping status, which resulted in eight subgroups: (1) exclusive daily smokers (2) exclusive non-daily smokers; (3-6) concurrent users (subdivided into four groups by each combination of daily/non-daily smoking and daily/non-daily vaping); (7) ex-smokers who vape; (8) ex-smokers not vaping. The analyses focused first on describing changes between groups from W1 to W2. Second, transition outcomes were assessed based on 
changes in smoking and vaping between W1 and W2. Transitions focused on smoking were: no change in smoking (continued smoking at the same frequency); decreased smoking; increased smoking; discontinued smoking; relapsed (ex-smokers at W1 who were smoking at W2). Transitions focused on vaping were: initiated vaping; switched from smoking to vaping. Overall, this study found that the vast majority of smokers were smoking 18 months later. Non-daily smokers were more likely than daily smokers to have discontinued smoking $(p<0.0001)$ and to have switched to exclusive vaping $(p=0.034)$. Exclusive non-daily smokers were more likely than exclusive daily smokers to have initiated vaping $(p=0.04)$. Among all W1 daily smokers, there were no differences in discontinued smoking between daily smokers who vaped (concurrent users) and exclusive daily smokers; however, concurrent users were more likely than exclusive daily smokers to have decreased to non-daily smoking $(p<0.001)$ or to have switched to vaping by W2 $(p<0.001)$. Among all W1 non-daily smokers, there were no significant differences in increased smoking or discontinued smoking between concurrent users or exclusive smokers. Most ex-smokers remained abstinent from smoking, and there was no difference in relapse back to smoking between those who vaped and those who did not.

Keywords: smoking; transitions; tobacco; nicotine vaping products; adults; e-cigarettes

\section{Introduction}

Cigarettes remain the most dangerous and commonly consumed nicotine product [1]. However, an increasing number of smokers are also using non-combusted tobacco/nicotine products, such as e-cigarettes, heated tobacco products, and snus [1-3]. The most popular and rapidly growing class of these non-combustible products are nicotine vaping products (NVPs, commonly known as e-cigarettes) [3]. Evidence suggests that completely substituting NVPs for combustible cigarettes greatly reduces users' exposure to numerous toxicants and carcinogens $[2,4,5]$.

Over the last decade, scientists, clinicians, advocates, and public health organizations have debated whether or not NVPs yield a net benefit to population health [2]. The key question with respect to smokers, is whether NVPs can help them transition away from cigarettes, and remain abstinent from smoking. A recent randomized trial found that among a sample of 886 adult smokers seeking help to quit smoking, significantly more smokers using an NVP quit smoking after one year $(18.0 \%)$ compared to smokers receiving nicotine replacement therapy (NRT) $(9.9 \%)$ [6]. Another randomized trial found that combination therapy of nicotine patches with a nicotine e-cigarette was associated with a modest improvement in continuous abstinence at six months $(7 \%)$ compared to NRT plus a nicotine-free e-cigarette ( $4 \%$ ) or NRT alone (2\%) [7]. However, findings from randomized trials may not be generalizable to whether NVPs are associated with reducing or discontinuing smoking in the manner in which they are used by smokers in the natural environment. It is important to understand the extent to which vaping can facilitate transitioning away from smoking in "real-world" settings, even as we recognize the challenges in making causal inferences with non- randomized trial study designs.

The increasing diversification of the NVP landscape has implications for understanding how product use changes over time (e.g., transitioning between combustible and non-combustible products), and longitudinal cohort studies are essential for assessing these transitions [8,9]. Examining patterns of smoking and vaping, especially exploring the nature of these transitions between cigarettes and NVPs over time, is a beginning point for describing transitioning in and out of smoking, and how vaping may play a role in these transitions.

Using the Population Assessment of Tobacco and Health (PATH) Study (a national population longitudinal study that tracks tobacco product use in a representative sample of adults in the United States (US)), Hyland et al. (2020) described patterns of tobacco/nicotine product use among current and former smokers across time [9]. The main categories included: persistent use (continued use 
across multiple time points), discontinued use (use to no use), relapse (stopped use and began use again), switching (changing between product types), and inconsistent use (back and forth between use and non-use). Evidence from the PATH Study has shown that the majority of cigarette smokers persist with smoking across time $[10,11]$, whereas transition behaviors among smokers initiating and persisting with NVP use (concurrent users) are more highly variable [11-13]. Moreover, continuing with/transitioning toward cigarettes, is more common than continuing with/transitioning toward NVPs. When it comes to differential transitions away from smoking by vaping status, Coleman et al. [12] found that among baseline concurrent users, daily vapers were more likely than non-daily vapers to report smoking abstinence at follow-up. Kasza et al. [13] found that vaping was positively associated with making attempts to quit, but was not associated with discontinuing smoking among attempters. However, some caution is required when interpreting population-based studies as NVP use is not randomized and there is reason to believe those vaping differ from those who are not. For example, concurrent users have been shown to be more dependent on nicotine than smokers who do not vape [14,15]. Further, baseline smokers who use NVPs could be viewed as "treatment failures" if they initiated NVP use to help them to stop smoking, but had not quit at the time of the follow-up survey.

In this current prospective cohort study, data from the ITC Four Country and Vaping Surveys (ITC $4 \mathrm{CV}$ ) were used to explore and describe behavioral transitions among smokers and recent ex-smokers in "real world" conditions at two time points (18 months apart) in four high-income countries, allowing us to examine transitions between smoking and vaping across a broader international context. Specifically, this study examined point prevalence states of smoking and vaping at baseline and follow-up among exclusive smokers (daily and non-daily/past-daily; no vaping), concurrent users (based on both smoking and vaping frequency), and recent ex-smokers (who either vaped or not). Exploratory analyses were also conducted to compare transition outcomes at follow-up between subgroups (based on baseline vaping or not): no change; decreased smoking (from daily to non-daily); increased smoking (from non-daily to daily); discontinued smoking; switched from smoking to vaping; initiated vaping. Relapse back to smoking was assessed for ex-smokers based on baseline vaping or not. Finally, we conducted difference-in-differences (DID) analyses by country (as NVP policies vary across these four countries), to test whether smokers who were vaping were more likely to decrease or discontinue smoking (compared to those who were not vaping). The authors did not have any preconceived hypotheses, as these exploratory analyses were based on findings from the transition estimates.

\section{Methods}

\subsection{Study Sample and Procedure}

The ITC 4CV Survey is a longitudinal cohort study that consists of four parallel online surveys conducted in Canada, the US, England, and Australia. In addition to respondents retained from the ITC Four Country Survey (the predecessor of ITC $4 \mathrm{CV}$ ), adults ( $\geq 18$ years) were recruited by commercial panel firms in each country at Wave 1 (W1: July-November 2016) as cigarette smokers (smoked at least 100 cigarettes in their lifetime and smoked at least monthly), recent ex-smokers (quit within $\leq 2$ years), or at-least-weekly NVP users (vapers). The sample in each country was designed to be as representative as possible of cigarette smokers, ex-smokers, and NVP users (e.g., by age, sex, and region). All W1 respondents were invited back to complete the Wave 2 (W2: February-July 2018) survey, and were included in the current study if they were at least monthly smokers or recent ex-smokers at W1 and completed W2. All those who were non-daily smokers at baseline must have smoked daily in the past to be considered for inclusion. All respondents provided consent, and all study procedures were approved by relevant research ethics boards. Further details about the ITC 4CV study can be found in the 2016 [16] and 2018 [17] technical reports, and in Thompson et al. [18]. 


\subsection{Measures}

The surveys, with original response options, can be found at the ITC Project website: https: //itcproject.org/surveys/. The following variables were used in the current study:

\subsubsection{Wave 1 (2016) Baseline Measures}

Sociodemographic variables: sociodemographic data were collected by the commercial panels and verified at the time of survey completion, including: age, gender, education, and country of residence.

Smoking and vaping status: respondents who reported smoking at least monthly (daily vs. non-daily) or recently quit smoking at baseline were included in this study. A parallel question was asked about NVP use and vaping frequency, and users were categorized as: current daily vapers, current non-daily vapers, or not currently vaping (those who were vaping less than monthly were considered non-vapers).

Time-in-sample (TIS): the analyses controlled for the time-in-sample (TIS), the number of waves that the respondent completed. TIS has been found to be related to differences in responses to a number of outcome variables in past ITC studies. Methodological details of TIS are presented elsewhere [19].

\subsubsection{Country NVP Regulations}

Country regulations covering NVPs have been previously summarized [20]. In brief, in 2016, the US allowed NVPs to be regulated and sold with few federal restrictions, England allowed NVPs to be sold and were regulated under the UK Tobacco and Related Products Regulations [21]. In contrast, NVPs were not permitted to be legally sold in Canada (with weak enforcement) or Australia (with strong enforcement). In 2018, the only major change in national regulatory policies was that the Canadian government passed the Tobacco and Vaping Products Act [22], which allowed NVPs to be regulated and sold as of May 2018.

\subsubsection{Classification of Point Prevalence Smoking and Vaping Status}

The respondents were categorized in terms of daily and non-daily smoking and vaping as described in Table 1. The categorization of concurrent users is according to the system developed by Borland et al. [23].

Table 1. Group classification of respondents' smoking and vaping status.

\begin{tabular}{cccc}
\hline$\#$ & Group Category & Smoking & Vaping \\
\hline \multicolumn{2}{c}{ Exclusive } & smokers & \\
1 & Exclusive daily smokers & Daily & None \\
2 & Exclusive non-daily smokers & Non-daily & None \\
Concurrent users & & \\
3 & Dual-daily users & Daily & Daily \\
4 & Predominant smokers & Daily & Non-daily \\
5 & Predominant vapers & Non-daily & Daily \\
6 & Concurrent non-daily users & Non-daily & Non-daily \\
Recent ex-smokers & & \\
7 & Ex-smokers who vape & None & Daily/Non-daily \\
8 & Ex-smokers not vaping & None & None \\
\hline
\end{tabular}

Note: Ex-smokers who vape (exclusive vapers) could not be subdivided by daily or non-daily vaping due to small sample sizes.

\subsubsection{Wave 2 (2018) Transition Outcomes}

Transition outcomes were determined by respondents' self-reported smoking and vaping statuses at W1 and W2. The following seven transition outcomes were deemed relevant to the research question, and were adapted from the approach described by Hyland et al. [9]. The transition outcomes focused only on smoking (A-E) were: (A) no change in smoking status (continued smoking at 
the same frequency), (B) decreased smoking, (C) increased smoking, (D) discontinued smoking, and (E) relapsed (ex-smokers at W1 who were smoking at W2). Transition outcomes focused on vaping (F and $G$ ) were: (F) initiated vaping; $(G)$ switched from smoking to vaping (a sub-analysis of (4) above).

\subsection{Statistical Analyses}

Unweighted frequencies were used to describe the respondents' baseline (W1) characteristics (Table 2). All subsequent analyses were weighted using longitudinal weights that were computed for all respondents. In brief, a raking algorithm [24] was used to calibrate the weights on smoking status, geographic region, and demographic measures (e.g., sex, age, ethnicity, and education). This calibration was done using benchmarks from national surveys from each of the respective countries. Generalized estimating equations (GEEs) using the predicted marginal standardization method [25] were used to generate regression models (PREDMARG) to estimate the point prevalence states of smoking and vaping at both time points.

There were three main sets of analyses conducted for this study. First, multivariable logistic regression models were used to descriptively examine within-person transitions between states of smoking and vaping (based on Table 1 group classifications: $1-8)$ among W1 daily smokers $(n=3983$; exclusive daily smokers, predominant smokers, dual-daily users), non-daily smokers ( $n=453$; exclusive non-daily smokers, concurrent non-daily users, predominant vapers); recent ex-smokers ( $n=580$; vaping or not vaping) (Table 3). These descriptive analyses focused on changes between groups that occurred in W2 (as well as remaining in the same state of smoking and/or vaping) and estimated point prevalence W2 estimates for the applicable transitions. The analyses for W1 daily smokers and recent ex-smokers controlled for age, country, education, and TIS (gender was not significant in the bivariate analyses and therefore was not used as a covariate in the larger model). Given the relatively small sample sizes, the analysis for W1 non-daily smokers used the same covariates as the previous model, with the exception of country. Additionally, groups 5 and 6 (predominant vapers and concurrent non-daily users) were combined at W2 due to small sample sizes.

The second set of analyses were exploratory in nature and compared outcomes between subgroups based on the seven transitions outlined in Section 2.2.4. The transition outcomes focused on smoking were: (A) no change (continued smoking at the same frequency)-(i) exclusive daily smokers vs. daily smokers who were vaping; (ii) exclusive non-daily smokers vs. non-daily smokers who were vaping; (B) decreased smoking from daily to non-daily-exclusive daily smokers vs. daily smokers who were vaping; $(C)$ increased smoking - exclusive non-daily smokers vs. non-daily smokers who were vaping; (D) discontinued smoking-(i) all daily smokers vs. all non-daily smokers; (ii) exclusive daily smokers vs. daily smokers who were vaping; (iii) exclusive non-daily smokers vs. non-daily smokers who were vaping; (iv) between the concurrent user groups; (E) relapsed back to smoking-ex-smokers who were vaping vs. ex-smokers who were not vaping. Transition outcomes focused on vaping were: $(\mathrm{F})$ initiated vaping - exclusive daily smokers vs. exclusive non-daily smokers; $(G)$ discontinued smoking and switched to vaping-(i) all daily smokers vs. all non-daily smokers; (ii) exclusive daily smokers vs. daily smokers who were vaping; (iii) exclusive non-daily smokers vs. non-daily smokers who were vaping; (iv) between the concurrent user groups (Table 4). The same covariates were used in the models as described above.

The third set of analyses tested differences by country and baseline NVP use (yes or no) for: (1) daily smokers who decreased to non-daily smoking or discontinued smoking between W1 and W2; (2) non-daily smokers discontinuing smoking between W1 and W2. These analyses examined if there were differences within countries, and if these differences differed between countries (using the DID method) [26]. The analyses controlled for age, education, TIS, and uptake of NVP use between W1 and W2 (regardless of vaping history). 
Table 2. Respondents' Baseline (2016) Characteristics.

\begin{tabular}{|c|c|c|c|c|c|}
\hline \multicolumn{2}{|c|}{ Characteristics, $n(\%)$} & \multirow{2}{*}{$\begin{array}{c}\begin{array}{c}\text { Exclusive Smokers } \\
n=3319(66.2 \%)\end{array} \\
636(19.2)\end{array}$} & \multirow{2}{*}{$\begin{array}{c}\begin{array}{c}\text { Concurrent Users } \\
\boldsymbol{n}=\mathbf{1 1 1 7}(\mathbf{2 2 . 3} \%)\end{array} \\
90(8.1)\end{array}$} & \multirow{2}{*}{$\begin{array}{c}\begin{array}{c}\text { Ex-Smokers } \\
n=580(\mathbf{1 1 . 6 \% )})\end{array} \\
93(16.0)\end{array}$} & \multirow{2}{*}{$\begin{array}{c}\text { Overall } \\
\mathbf{N}=5016 \\
819(16.3)\end{array}$} \\
\hline Country & Australia & & & & \\
\hline & Canada & $972(29.3)$ & $439(39.3)$ & $187(32.2)$ & $1598(31.9)$ \\
\hline & England & $1040(31.3)$ & $359(32.1)$ & $151(26.0)$ & $1550(30.9)$ \\
\hline & United States & $671(20.2)$ & $229(20.5)$ & 149 (25.7) & 1049 (20.9) \\
\hline \multirow[t]{2}{*}{ Sex } & Male & $1592(48.0)$ & $592(53.0)$ & $263(45.3)$ & $2447(48.8)$ \\
\hline & Female & $1727(52.0)$ & $525(47.0)$ & 317 (54.7) & $2569(51.2)$ \\
\hline Age & Mean (SD) & $50.9(13.7)$ & $44.2(15.2)$ & $49.6(14.7)$ & $49.3(14.5)$ \\
\hline \multirow[t]{4}{*}{ Age group } & $18-24$ & $184(5.5)$ & $162(14.5)$ & $30(5.2)$ & $376(7.5)$ \\
\hline & $25-39$ & $536(16.2)$ & $298(26.7)$ & $126(21.7)$ & $960(19.1)$ \\
\hline & $40-54$ & $1097(32.0)$ & $331(29.6)$ & $177(30.5)$ & $1605(32.0)$ \\
\hline & $55+$ & $1502(41.4)$ & $326(29.2)$ & $247(42.6)$ & $2075(41.4)$ \\
\hline \multirow[t]{4}{*}{ Education level } & Low & $1154(34.8)$ & $310(27.8)$ & $171(29.5)$ & $1635(32.6)$ \\
\hline & Moderate & $1350(40.7)$ & $445(39.8)$ & $250(43.1)$ & $2045(40.8)$ \\
\hline & High & $789(23.8)$ & $351(31.4)$ & $156(26.9)$ & $1296(25.8)$ \\
\hline & Not reported & $26(0.8)$ & $11(1.0)$ & $3(0.5)$ & $40(0.8)$ \\
\hline \multirow[t]{3}{*}{ Smoking Status } & Daily smoking & $3063(92.3)$ & $920(82.4)$ & - & $3983(79.4)$ \\
\hline & Non-daily smoking & $256(7.7)$ & 197 (17.6) & - & $453(9.0)$ \\
\hline & Recent ex-smoker & - & - & $580(100.0)$ & $580(11.6)$ \\
\hline \multirow[t]{3}{*}{ Vaping Status } & Daily vaping & - & $410(36.7)$ & $109(18.8)$ & $519(10.4)$ \\
\hline & Non-daily vaping & - & 707 (63.3) & $27(4.7)$ & $734(14.6)$ \\
\hline & No current vaping & $3319(100.0)$ & - & $444(76.6)$ & $3763(75.0)$ \\
\hline
\end{tabular}

Data are unweighted in order to describe the sample used in the analyses; SD: standard deviation. 
Table 3. User group transitions in smoking and vaping among daily smokers, non-daily smokers, and recent ex-smokers.

\begin{tabular}{|c|c|c|c|c|c|c|c|c|c|c|c|c|}
\hline & \multirow[b]{4}{*}{ Wave 1} & & \multicolumn{10}{|c|}{ Wave 2} \\
\hline & & & \multicolumn{7}{|c|}{ Smoking } & \multirow{2}{*}{\multicolumn{3}{|c|}{ Not Smoking }} \\
\hline & & & \multicolumn{2}{|c|}{ Exclusive Smoking } & \multicolumn{3}{|c|}{ Concurrent Use } & \multicolumn{2}{|c|}{ Total Smoking } & & & \\
\hline & & & $\begin{array}{c}\text { Exclusive } \\
\text { Daily } \\
\text { Smokers } \\
\text { (1) }\end{array}$ & $\begin{array}{c}\text { Exclusive } \\
\text { Non-Daily } \\
\text { Smokers } \\
\text { (2) }\end{array}$ & $\begin{array}{l}\text { Dual-Daily } \\
\text { Users } \\
\text { (3) }\end{array}$ & $\begin{array}{l}\text { Predominant } \\
\text { Smokers } \\
\text { (4) }\end{array}$ & $\begin{array}{l}\text { Predominant } \\
\text { Vapers and } \\
\text { Concurrent } \\
\text { Non-Daily } \\
\text { Users* } \\
(5+6)\end{array}$ & $\begin{array}{c}\text { Total Daily } \\
\text { Smokers } \\
(1+3+4)\end{array}$ & $\begin{array}{c}\text { Total } \\
\text { Non-Daily } \\
\text { Smokers } \\
(2+5+6)\end{array}$ & $\begin{array}{c}\text { Ex-Smokers } \\
\text { Who Vape } \\
\text { (7) }\end{array}$ & $\begin{array}{c}\text { Ex-Smokers } \\
\text { Not Vaping } \\
\text { (8) }\end{array}$ & $\begin{array}{c}\text { Total } \\
\text { Discontinued } \\
\text { Smoking } \\
(7+8)\end{array}$ \\
\hline \multicolumn{13}{|c|}{ Exclusive smokers $(n=3319)$} \\
\hline \multirow{2}{*}{1} & \multirow{2}{*}{$\begin{array}{l}\text { Exclusive daily } \\
\text { smokers }(n=3063)\end{array}$} & $\%$ & 71.4 & 3.0 & 3.7 & 8.9 & 1.5 & 84.0 & 4.5 & 2.6 & 9.0 & 11.6 \\
\hline & & $n$ & 2296 & 79 & 94 & 223 & 37 & 2613 & 116 & 73 & 261 & 334 \\
\hline \multirow{2}{*}{2} & \multirow{2}{*}{$\begin{array}{l}\text { Exclusive non-daily } \\
\text { smokers }(n=256)\end{array}$} & $\%$ & 21.1 & 31.2 & 4.2 & 8.0 & 8.4 & 33.3 & 39.6 & 4.8 & 22.5 & 27.3 \\
\hline & & $n$ & 55 & 84 & 7 & 16 & $\begin{array}{l}0.4 \\
22\end{array}$ & 78 & 106 & 11 & 61 & 72 \\
\hline \multicolumn{13}{|c|}{ Concurrent users $(n=1117)$} \\
\hline \multirow{2}{*}{3} & \multirow{2}{*}{$\begin{array}{l}\text { Dual-daily users } \\
(n=322)\end{array}$} & $\%$ & 21.4 & 1.5 & 38.5 & 18.6 & 8.3 & 78.5 & 9.8 & 9.5 & 2.2 & 11.7 \\
\hline & & $n$ & 46 & 3 & 129 & 76 & 34 & 251 & 37 & 25 & 9 & 34 \\
\hline \multirow{2}{*}{4} & \multirow{2}{*}{$\begin{array}{l}\text { Predominant } \\
\text { smokers }(n=598)\end{array}$} & $\%$ & 42.2 & 2.7 & 14.9 & 25.4 & 5.4 & 82.5 & 8.1 & 5.0 & 4.5 & 9.5 \\
\hline & & $n$ & 216 & 16 & 89 & 187 & 40 & 492 & 56 & 26 & 24 & 50 \\
\hline \multirow{2}{*}{5} & \multirow{2}{*}{$\begin{array}{l}\text { Predominant } \\
\text { vapers }(n=88)\end{array}$} & $\%$ & 4.2 & 1.7 & 12.0 & 18.7 & 39.7 & 34.9 & 41.4 & 18.0 & 5.8 & 23.8 \\
\hline & & $n$ & 6 & 3 & 17 & 12 & 29 & 35 & 32 & 15 & 6 & 21 \\
\hline \multirow{4}{*}{6} & \multirow{3}{*}{$\begin{array}{l}\text { Concurrent } \\
\text { non-daily users } \\
(n=109)\end{array}$} & $\%$ & 5.3 & 9.9 & 7.1 & 9.0 & 51.2 & 21.4 & 61.1 & 8.2 & 9.4 & 17.6 \\
\hline & & $n$ & 6 & 8 & 10 & 15 & 48 & 31 & 56 & 10 & 12 & 22 \\
\hline & & & \multicolumn{7}{|c|}{ Recent ex-smokers ${ }^{\dagger}(n=580)$} & & & \\
\hline & \multicolumn{9}{|c|}{ Relapsed (exclusive smoking or concurrent use) } & \multicolumn{3}{|c|}{ Continued smoking abstinence } \\
\hline \multirow{2}{*}{7} & \multirow{2}{*}{$\begin{array}{l}\text { Ex-smokers who } \\
\text { vape }(n=136)\end{array}$} & $\%$ & \multirow{2}{*}{\multicolumn{2}{|c|}{$\begin{array}{c}4.4(1+2) \\
2\end{array}$}} & \multirow{2}{*}{\multicolumn{3}{|c|}{$\begin{array}{c}8.1(3-6) \\
8\end{array}$}} & \multirow{2}{*}{\multicolumn{2}{|c|}{$\begin{array}{c}12.5(1-6) \\
10\end{array}$}} & 67.3 & 20.2 & 87.5 \\
\hline & & $n$ & & & & & & & & 94 & 32 & 126 \\
\hline \multirow{2}{*}{8} & \multirow{2}{*}{$\begin{array}{l}\text { Ex-smokers not } \\
\text { vaping }(n=444)\end{array}$} & $\%$ & 11.3 & $(1+2)$ & & $2.0(3-6)$ & & 13.3 & $(-6)$ & 4.6 & 82.1 & 86.7 \\
\hline & & $n$ & & 47 & & 8 & & & & 21 & 368 & 389 \\
\hline
\end{tabular}


Table 3. Cont.

\begin{tabular}{|c|c|c|c|c|c|c|c|c|c|c|c|}
\hline Row Totals & & & & & & & & & & & \\
\hline All daily smokers $(n=3983)$ & $\%$ & 67.2 & 2.9 & 5.8 & 10.6 & 2.1 & 83.6 & 5.0 & 2.9 & 8.4 & 11.3 \\
\hline $\begin{array}{l}\text { All non-daily smokers } \\
(n=453)\end{array}$ & $\%$ & 17.7 & 26.1 & 5.5 & 9.2 & 15.5 & 32.4 & 41.6 & 6.5 & 19.6 & 26.1 \\
\hline $\begin{array}{l}\quad \text { All recent ex-smokers } \\
(n=580)\end{array}$ & $\%$ & \multicolumn{2}{|c|}{$10.5(1+2)$} & \multicolumn{3}{|c|}{$2.8(3-6)$} & \multicolumn{2}{|c|}{$13.3(1-6)$} & 12.2 & 74.6 & 86.8 \\
\hline
\end{tabular}

Data are weighted and adjusted with covariates. The numbering system (1-8) corresponds to Table $1 .{ }^{*}$ Groups 5 and 6 were merged at Wave 2 due to small sample sizes; ${ }^{\dagger}$ Recent ex-smokers could not be further subdivided at Wave 2 due to small sample sizes. Bold: Weighted estimates are bolded; Italics: sample sizes are italicized. 
Table 4. Wave 1 to Wave 2 transition subgroup comparisons: progression towards discontinuing smoking among daily and non-daily smokers and relapse among ex-smokers based on baseline frequency of smoking and vaping.

\begin{tabular}{|c|c|c|c|c|c|}
\hline $\begin{array}{c}\text { Wave } 1 \text { to Wave } 2 \\
\text { Transitions }\end{array}$ & Transition Code * & Comparisons betv & Wave 1 Subgroups & OR & $95 \%$ CI \\
\hline \multirow{3}{*}{$\begin{array}{l}\text { No change in smoking } \\
\text { frequency (daily) }\end{array}$} & \multirow{3}{*}{ A (i) } & 1 & Exclusive daily smokers & \multicolumn{2}{|c|}{ Reference } \\
\hline & & 3 vs. 1 & Dual-daily users & 0.72 & 0.49-1.05 \\
\hline & & 4 vs. 1 & Predominant smokers & 0.91 & $0.67-1.24$ \\
\hline \multirow{3}{*}{$\begin{array}{l}\text { No change in smoking } \\
\text { frequency (non-daily) }\end{array}$} & \multirow{3}{*}{ A (ii) } & 2 & Exclusive non-daily & \multicolumn{2}{|c|}{ Reference } \\
\hline & & 5 vs. 2 & $\begin{array}{l}\text { smokers } \\
\text { Predominant vapers }\end{array}$ & 0.99 & $0.51-1.90$ \\
\hline & & 6 vs. 2 & $\begin{array}{c}\text { Concurrent non-daily } \\
\text { users }\end{array}$ & 2.60 & $1.45-4.67$ \\
\hline \multirow{3}{*}{$\begin{array}{l}\text { Decreased smoking from } \\
\text { daily to non-daily }\end{array}$} & \multirow{3}{*}{ B } & 1 & Exclusive daily smokers & \multicolumn{2}{|c|}{ Reference } \\
\hline & & 3 vs. 1 & Dual-daily users & 2.41 & $1.42-4.10$ \\
\hline & & 4 vs. 1 & Predominant smokers & 1.90 & $1.23-2.95$ \\
\hline \multirow{3}{*}{$\begin{array}{l}\text { Increased smoking from } \\
\text { non-daily to daily }\end{array}$} & \multirow{3}{*}{$\mathrm{C}$} & 2 & Exclusive non-daily & \multicolumn{2}{|c|}{ Reference } \\
\hline & & 5 vs. 2 & $\begin{array}{l}\text { smokers } \\
\text { Predominant vapers }\end{array}$ & 1.12 & $0.58-2.15$ \\
\hline & & 6 vs. 2 & $\begin{array}{c}\text { Concurrent non-daily } \\
\text { users }\end{array}$ & 0.54 & $0.28-1.04$ \\
\hline \multirow{12}{*}{ Discontinued smoking } & \multirow{2}{*}{$\mathrm{D}(\mathrm{i})$} & $1+3+4$ & Daily smokers & \multicolumn{2}{|c|}{ Reference } \\
\hline & & $2+5+6$ vs. $1+3+4$ & Non-daily smokers & 2.48 & $1.80-3.43$ \\
\hline & \multirow{3}{*}{ D (ii) } & 1 & Exclusive daily smokers & Reference & \\
\hline & & 3 vs. 1 & Dual-daily users & 0.87 & $0.53-1.41$ \\
\hline & & 4 vs. 1 & Predominant smokers & 0.75 & $0.50-1.12$ \\
\hline & \multirow{3}{*}{$\mathrm{D}$ (iii) } & 2 & $\begin{array}{l}\text { Exclusive non-daily } \\
\text { smokers }\end{array}$ & Reference & \\
\hline & & 5 vs. 2 & Predominant vapers & 0.89 & $0.43-1.83$ \\
\hline & & 6 vs. 2 & $\begin{array}{c}\text { Concurrent non-daily } \\
\text { users }\end{array}$ & 0.55 & $0.29-1.06$ \\
\hline & \multirow{4}{*}{$\mathrm{D}$ (iv) } & 3 & Dual-daily users & Reference & \\
\hline & & 4 vs. 3 & Predominant smokers & 0.83 & $0.48-1.45$ \\
\hline & & 5 vs. 3 & Predominant vapers & 2.40 & $1.07-5.35$ \\
\hline & & 6 vs. 3 & $\begin{array}{c}\text { Concurrent non-daily } \\
\text { users }\end{array}$ & 1.86 & $0.92-3.75$ \\
\hline
\end{tabular}


Table 4. Cont

\begin{tabular}{|c|c|c|c|c|c|}
\hline \multirow[b]{2}{*}{ Relapsed back to smoking } & \multirow[b]{2}{*}{$\mathrm{E}$} & \multirow{2}{*}{$\begin{array}{c}7 \\
8 \text { vs. } 7\end{array}$} & \multirow{2}{*}{$\begin{array}{c}\text { Ex-smokers who vape } \\
\text { Ex-smokers who don't } \\
\text { vape }\end{array}$} & \multicolumn{2}{|c|}{ Reference } \\
\hline & & & & 1.04 & $0.36-3.03$ \\
\hline \multirow[b]{2}{*}{ Initiated Vaping } & \multirow[b]{2}{*}{$\mathrm{F}$} & 1 & \multirow{2}{*}{$\begin{array}{l}\text { Exclusive daily smokers } \\
\text { Exclusive non-daily } \\
\text { smokers }\end{array}$} & Reference & \\
\hline & & 2 vs. 1 & & 1.52 & $1.02-2.25$ \\
\hline \multirow{12}{*}{$\begin{array}{l}\text { Discontinued smoking and } \\
\text { switched to vaping }\end{array}$} & \multirow[b]{2}{*}{ G (i) } & $1+3+4$ & Daily smokers & \multicolumn{2}{|c|}{ Reference } \\
\hline & & $2+5+6$ vs. $1+3+4$ & Non-daily smokers & 2.21 & $1.25-3.91$ \\
\hline & \multirow{3}{*}{ G (ii) } & 1 & Exclusive daily smokers & \multicolumn{2}{|c|}{ Reference } \\
\hline & & 3 vs. 1 & Dual-daily users & 3.55 & $1.90-6.64$ \\
\hline & & 4 vs. 1 & Predominant smokers & 1.88 & $1.09-3.23$ \\
\hline & \multirow{3}{*}{ G (iii) } & 2 & $\begin{array}{l}\text { Exclusive non-daily } \\
\text { smokers }\end{array}$ & \multicolumn{2}{|c|}{ Reference } \\
\hline & & 5 vs. 2 & Predominant vapers & 4.99 & $1.82-13.72$ \\
\hline & & 6 vs. 2 & $\begin{array}{l}\text { Concurrent non-daily } \\
\text { users }\end{array}$ & 1.81 & $0.61-5.35$ \\
\hline & \multirow{4}{*}{ G (iv) } & 3 & Dual-daily users & Reference & \\
\hline & & 4 vs. 3 & Predominant smokers & 0.52 & $0.27-1.01$ \\
\hline & & 5 vs. 3 & Predominant vapers & 2.25 & $0.89-5.70$ \\
\hline & & 6 vs. 3 & $\begin{array}{c}\text { Concurrent non-daily } \\
\text { users }\end{array}$ & 1.09 & $0.44-2.72$ \\
\hline
\end{tabular}

Data are weighted and adjusted with covariates. The "group comparison" numbering system refers to Table 1. * Transition codes refer to the group comparisons as described for the second analyses in the Statistical Analyses Section 2.3. Control groups were exclusive smokers (compared to concurrent users). Among concurrent users, dual-daily users were used as the control group (the group with the most frequent smoking and vaping); vs: versus. Bold odds ratios indicate significance. OR: Odds ratio; CI: Confidence interval. 
Statistical significance and confidence intervals were computed at the 95\% confidence level. Analyses were conducted in SAS-Callable SUDAAN (V.11; RTI International, Research Triangle Park, NC, USA).

\subsection{Ethics approval}

Study questionnaires and materials were reviewed and provided clearance by Research Ethics Committees at the following institutions: University of Waterloo (Canada, ORE\#20803/30570, ORE\#21609/30878), King's College London, UK (RESCM-17/18-2240), Cancer Council Victoria, Australia (HREC1603), University of Queensland, Australia (2016000330/HREC1603); and Medical University of South Carolina (waived due to minimal risk).

\section{Results}

Overall, 5632 W1 respondents in the larger cohort study were followed up and had complete data at W2. Those who had never smoked $(n=24)$, smoked less than monthly $(n=255)$, were never daily smokers $(n=136)$, or quit smoking more than 2 years ago $(n=201)$ were excluded for this study, thus resulting in 5016 respondents being included in the analyses: exclusive (at least monthly) smokers $(n=3319)$, concurrent users (concurrently smoke and vape at least monthly, $n=1117$ ), and recent ex-smokers ( $n=580$ : of whom 33.5\% quit smoking within the last 6 months, $25.0 \%$ between 7 and 12 months, and $41.5 \%$ between 1 and 2 years ago). Respondent baseline characteristics are presented in Table 2.

\subsection{User Group Transitions among Daily Smokers and Non-Daily Smokers: Point Prevalence W2 Estimates}

Table 3 shows the transitions between the eight subgroups between W1 and W2. Transitions are briefly described below:

\subsection{Transitions among Daily Smokers}

W1 exclusive daily smokers: At W2: 71.4\% did not change (remained exclusive daily smokers and not vaping), 3.0\% decreased to non-daily smoking, $14.1 \%$ became concurrent users, and $11.6 \%$ were ex-smokers (discontinued smoking).

W1 predominant smokers: At W2: $25.4 \%$ did not change (remained smoking daily and vaping less than daily), $42.2 \%$ became exclusive daily smokers, $2.7 \%$ became exclusive non-daily smokers, 9.5\% were ex-smokers (discontinued smoking).

W1 dual-daily users: At W2: 38.5\% did not change (remained using both products daily), $21.4 \%$ became exclusive daily smokers, $1.5 \%$ became exclusive non-daily smokers, and $11.7 \%$ were ex-smokers (discontinued smoking).

\subsection{Transitions among Non-Daily Smokers}

W1 exclusive non-daily smokers: At W2: 31.2\% did not change (remained exclusive non-daily smokers), $20.6 \%$ became concurrent users, $21.1 \%$ became exclusive daily smokers, and $27.3 \%$ were ex-smokers (discontinued smoking).

W1 predominant vapers: At W2: $39.7 \%$ did not change (remained vaping daily and smoking non-daily), $4.2 \%$ became exclusive daily smokers, $1.7 \%$ became exclusive non-daily smokers, and $23.8 \%$ were ex-smokers (discontinued smoking).

W1 concurrent non-daily users: At W2: $51.2 \%$ did not change (remained using both products on a non-daily basis), 5.3\% became exclusive daily smokers, 9.9\% became exclusive non-daily smokers, and $17.6 \%$ were ex-smokers (discontinued smoking). 


\subsection{Transitions among Recent Ex-Smokers}

W1 recent ex-smokers who vaped: $67.3 \%$ did not change (were still exclusively vaping and not smoking), $4.4 \%$ were exclusively smoking, and $8.1 \%$ became concurrent users.

W1 recent ex-smokers who did not vape: $82.1 \%$ did not change (remained abstinent from smoking and vaping), $11.3 \%$ were exclusively smoking, and $2.0 \%$ became concurrent users.

\subsection{Wave 1 to Wave 2 Transition Subgroup Comparisons}

Table 4 presents the subgroup comparisons (including the transition code) for each of the seven transition outcomes. The main subgroup comparisons are outlined below.

\subsection{Comparisons between Wave 1 Daily and Non-Daily Smokers}

Non-daily smokers were more likely than daily smokers to have discontinued smoking (transition code: $D(i))$ or to have switched to vaping (G(i)). Exclusive non-daily smokers were more likely than exclusive daily smokers to have initiated vaping between $\mathrm{W} 1$ and $\mathrm{W} 2$ (F).

\subsection{Comparisons between Wave 1 Daily Smokers: Exclusive Daily Smokers vs. Concurrent Users}

Decreasing smoking differed between exclusive smokers and concurrent users, where predominant smokers and dual-daily users were significantly more likely than exclusive daily smokers to have reduced to non-daily smoking by W2 (A(i)). There were no differences between exclusive daily smokers and concurrent users in discontinuing smoking (D(ii)). Among W1 daily smokers, concurrent users were more likely than exclusive smokers to have discontinued smoking by switching to vaping ( $G(i i))$.

\subsection{Comparisons between Wave 1 Non-daily Smokers: Exclusive Non-daily Smokers vs. Concurrent Users}

There were no significant differences in increased smoking $(C)$ or discontinued smoking (D(iii) between exclusive non-daily smokers and concurrent users.

\subsection{Comparisons between Wave 1 Concurrent Users}

Overall, $59.4 \%$ of W1 concurrent users were still concurrently using both products at W2 (data not shown in tables). With regard to subgroup comparisons (dual-daily users were used as the control group: the group with the most frequent smoking and vaping frequency), predominant vapers were more likely than dual-daily users to have discontinued smoking at W2 (D(iv)), but there was no statistical difference between these groups for having switched to vaping (G(iv)), likely owing to the small sample size of predominant vapers (resulting in a large 95\% confidence interval). There were no other differences.

\subsection{Comparisons between Recent Ex-smokers, Vapers and Non-Vapers:}

There were no differences in the relapse rates back to smoking between vapers and non-vapers (E). However, significantly fewer ex-smoking vapers transitioned to exclusive smoking compared to non-vapers $(p=0.045$, shown in Table 3$)$.

\subsection{Country Differences}

Table 5 presents daily smokers' and non-daily smokers' progression away from smoking by country and vaping status. In brief, there were considerable similarities between countries, but there were some differences: (1) daily smokers who vaped were more likely to have decreased from daily to non-daily smoking than smokers who did not vape in Canada $(p=0.002)$ and England $(p=0.03)$. This was not statistically significant in the US or Australia, although trended in the same direction in the US $(p=0.06)$; (2) daily smokers who vaped were less likely than those who did not vape to discontinue smoking in Canada $(p=0.003)$. This was not found for Australia, the US or England; (3) non-daily smokers who vaped were significantly less likely to have discontinued smoking compared to those who did not vape in Australia ( $p$ <.001) (this association was not found in the other three countries); (4) cross-country analyses showed that Australia had a larger difference for discontinued smoking between vapers and non-vapers compared to Canada $(p=0.003)$ and England $(p=0.018)$. 
Table 5. Country comparisons among daily smokers and non-daily smokers (past-daily smokers) and reduced smoking and discontinued smoking at Wave 2 (2018) between those who were vaping and not vaping at Wave 1 (2016).

\begin{tabular}{|c|c|c|c|c|c|c|c|c|c|c|c|c|c|c|}
\hline \multirow{2}{*}{$\begin{array}{c}\text { W1 } \\
\text { Smoking } \\
\text { Status }\end{array}$} & \multirow{2}{*}{$\begin{array}{c}\text { W1 } \\
\text { Vaping }\end{array}$} & \multirow{2}{*}{$\begin{array}{l}\text { W2 Smoking } \\
\text { Status }\end{array}$} & \multicolumn{3}{|c|}{ Canada $(n=1411)$} & \multicolumn{3}{|c|}{ United States $(n=900)$} & \multicolumn{3}{|c|}{ England ( $n=1399$ ) } & \multicolumn{3}{|c|}{ Australia $(n=726)$} \\
\hline & & & $n$ & $\%$ & $95 \%$ CI & $n$ & $\%$ & $95 \%$ CI & $n$ & $\%$ & $95 \% \mathrm{CI}$ & $n$ & $\%$ & $95 \%$ CI \\
\hline \multirow{6}{*}{$\begin{array}{c}\text { Daily } \\
n=3983\end{array}$} & Yes & Remained & 288 & 81.0 & $75.2-85.7$ & 134 & 66.1 & $56.3-74.7$ & 255 & 82.8 & $76.3-87.8$ & 66 & 83.0 & $67.9-91.9$ \\
\hline & No & daily & 731 & 82.7 & $79.5-85.4$ & 519 & 76.7 & $70.4-82.0$ & 834 & 88.4 & $85.3-90.9$ & 529 & 85.5 & $81.1-89.0$ \\
\hline & Yes & Reduced to & 43 & $12.6 *$ & $8.7-17.9$ & 23 & $16.2^{ \pm}$ & $9.9-25.5$ & 21 & $7.5^{+}$ & $4.4-12.6$ & 6 & 6.5 & $1.3-27.5$ \\
\hline & No & non-daily & 37 & 4.9 & $3.4-7.0$ & 28 & 8.4 & $5.2-13.5$ & 33 & 2.9 & $1.7-4.8$ & 18 & 3.2 & $1.7-5.9$ \\
\hline & Yes & Discontinued & 24 & $6.4^{*}$ & $3.9-10.3$ & 24 & 17.7 & $11.3-26.5$ & 26 & 9.7 & $6.0-15.4$ & 10 & 10.5 & $4.8-21.6$ \\
\hline & No & smoking & 104 & 12.4 & $10.1-15.2$ & 68 & 14.9 & $10.5-20.7$ & 99 & 8.8 & $6.6-11.5$ & 63 & 11.3 & $8.2-15.5$ \\
\hline \multirow{4}{*}{$\begin{array}{l}\text { Non-daily } \\
n=453\end{array}$} & Yes & Remained & 61 & 76.8 & $64.7-85.7$ & 39 & 75.5 & $52.8-89.4$ & 47 & 86.6 & $72.0-94.2$ & 7 & 98.7 & $88.6-99.9$ \\
\hline & No & smoking & 73 & 77.1 & $65.7-78.9$ & 37 & 54.5 & $34.6-73.0$ & 57 & 80.1 & $66.6-89.0$ & 17 & 60.2 & $38.7-78.4$ \\
\hline & Yes & Discontinued & 23 & 23.2 & $14.3-35.3$ & 9 & 24.5 & $10.6-47.2$ & 10 & 13.4 & $5.8-28.0$ & 1 & $1.3^{*}$ & $0.1-11.4$ \\
\hline & No & smoking & 27 & 22.9 & $15.1-33.0$ & 19 & 45.5 & $27.0-65.4$ & 17 & 19.9 & $11.0-33.4$ & 9 & $39.8^{*}$ & $21.6-61.3$ \\
\hline
\end{tabular}

${ }^{*} p<0.001 ;{ }^{\dagger} p<0.05 ;{ }^{ \pm} p<0.1$. The analyses adjusted for age, education, TIS, and uptake of NVP use between W1 and W2 (regardless of vaping history). Caution is warranted in interpreting any differences within or between countries due to small sample sizes in some cases. 


\section{Discussion}

This study is a descriptive analysis using a representative sample of smokers and ex-smokers, and described changes in smoking and vaping over 18 months. This study has also offered some insight into whether certain smoking and vaping subgroups differed in their smoking and vaping behaviors. Overall, this study found that the vast majority of smokers were smoking 18 months later, thus reflecting the high level of stability of this behavior. We found that non-daily smokers were more likely than daily smokers to have discontinued smoking at follow-up ( $26.1 \%$ vs. $11.3 \%$ respectively), which is consistent with non-daily smokers being less nicotine dependent than daily smokers [15]. With regard to vaping behaviors, a lower proportion of exclusive daily smokers than exclusive non-daily smokers initiated vaping between baseline and follow-up ( $16.7 \%$ vs. $25.4 \%$, respectively). Daily smokers were less likely than non-daily smokers to switch to exclusive vaping ( $2.9 \%$ vs. $6.5 \%$, respectively). When comparing exclusive smokers to concurrent users, daily smokers who were vaping at baseline (concurrent use) were more likely than exclusive daily smokers to have decreased smoking (from daily to non-daily); however, we found that concurrent use at baseline was not associated with discontinued smoking for either daily or non-daily smokers. About one-third of non-daily smokers increased smoking (to daily), and there were no significant differences between non-daily smokers who vaped or did not vape. The majority of ex-smokers in this study remained abstinent from smoking $(86.8 \%)$, and there were no differences in relapse between vapers and non-vapers, but baseline exclusive vapers were less likely than non-vapers to be exclusively smoking at follow-up ( $4.4 \%$ vs. $11.3 \%$, respectively).

These findings suggest that smokers with established concurrent use were not more likely to discontinue smoking compared to those not vaping. This was more evident for daily smokers, who are more highly addicted to nicotine than non-daily smokers [15,27-30]. In interpreting the results, it must be noted that concurrent users are potentially more highly addicted to nicotine than exclusive smokers [23]. For example, Strong et al. [15] examined indicators of tobacco dependence across a range of tobacco products and demonstrated that concurrent users of cigarettes and NVPs had the highest mean dependence scores. This could suggest that the unassisted quit rates in such smokers may be lower than for non-vapers, so it remains possible that vaping has equalized this imbalance, rather than having no effect on cessation. The finding of more reduction is consistent with this explanation. It is also important to note that while some NVP users are vaping to quit smoking, some are vaping for other reasons which is why they sustained continued smoking [31]. In this study, among baseline smokers who also vaped, $46 \%$ planned to quit smoking within 6 months, 30\% planned to quit in the future, but beyond 6 months, with the remaining $24 \%$ reporting that they did not know or did not plan on quitting, suggesting low motivation to quit smoking among many of the concurrent users. This is further supported by reasons that respondents gave for vaping, with $60 \%$ reporting that vaping may help them quit smoking, while $45 \%$ reported using an NVP for reasons other than to quit smoking (data not shown). This study, however, was not examining specific cessation attempts, but rather the naturalistic changes in nicotine product use over a period of time. Regardless, it is clear that the rates of transitioning away from smoking remain unacceptably low, and perhaps current vaping tools at best bring the likelihood of quitting up to comparable levels of less dependent smokers.

The findings of our international study are consistent with the findings of the US PATH transition studies, and other observational studies, in that most smokers remain in a persistent state of cigarette use across time, particularly the daily smokers [10,11,32-34]. For example, the vast majority of smokers in the PATH Study continue to smoke over time [10,11,35], and daily smoking was shown to be inversely associated with smoking abstinence [35]. Our findings are also consistent with the findings of a United Kingdom study (2016-2017) [33], in which 86\% of exclusive smokers were still smoking at follow-up.

The majority of ex-smokers in our study remained abstinent from smoking, and among those who relapsed, there was no difference between vapers and non-vapers. Few other studies have examined the role of NVPs in smoking relapse. One study found that vaping may be protective against relapse [14], but similar to our study, Brose et al. [36] reported that there was no difference in relapse 
between exclusive daily vapers compared to those who were not vaping. However, they did find that ex-smokers who vaped infrequently had a higher probability of relapse, suggesting that this group of ex-smokers were not vaping enough to satisfy their cravings for nicotine. More research is warranted to explore how vaping may help with smoking abstinence and prevent relapse, particularly among those who may have quit for a short period of time and may be more likely to relapse compared to those who have quit for longer periods of time [37,38].

This study extends our knowledge of smoking and vaping transitions to multiple countries, and showed, for the most part, considerable similarities. However, there were some differences. For example, in Canada and England, daily smokers who also vaped were more likely to transition to non-daily smoking (compared to exclusive smokers). There was a trend in the same direction for the US and Australia; however, small sample sizes may have limited the power to detect a significant difference. Disconcertingly, among daily smokers in Canada, discontinuation of smoking was less likely for those who vaped than for those who did not vape. The estimates for England the US were slightly in the opposite direction, and were equivalent in Australia. An unexpected finding was that England did not have a higher proportion of vapers discontinuing smoking compared to the other countries considering that England has the most supportive harm reduction policies $[39,40]$, whereas the other three countries have not taken the same approach. One unsurprising finding was that smokers from Australia (where NVPs are strictly prohibited) had very low rates of reducing smoking or discontinuing smoking among NVP users. This may be due to uncertainty of supply, or of the demands of needing to break laws to vape, acting as a disincentive to persist with vaping. Thus, the lower levels of vaping in Australia are likely related to the more restrictive laws about selling nicotine-containing e-liquids [41].

This study has several strengths. First, it is a large cohort study spanning across four countries, with differing NVP regulatory policies. Second, varying patterns in smoking and vaping frequencies over time were assessed, which is essential for characterizing daily nicotine users from non-daily nicotine users as they differ considerably (e.g., with regard to attitudes towards smoking and vaping, interest in quitting smoking, and nicotine dependence) [23]. There are, however, some limitations, therefore the findings from this paper should be interpreted with some caution. First, we only had two measurement periods, therefore we have only provided a snapshot of transition behaviors at two points in time, with no information about the intervening period (e.g., we did not explore actions taken between surveys such as use of NVPs for any quit attempts). Second, like all observational studies, vaping status was self-selected, not randomly allocated, so causal models could not be tested. Third, our sample design does not allow us to compute meaningful prevalence estimates for smoking or vaping (owing to the fact that we do not have a probability sample of the general population), thus we could not make assumptions about changes in smoking or vaping rates. Studies with a sample design involving a probability of the general population, such as the PATH Study [11], can provide prevalence estimates in addition to transitions. Fourth, some subgroups were small, thus limiting the power to detect significant differences, particularly when the sample was subdivided by country. Finally, we did not attempt to explore possible baseline differences between those in the various Wave 1 use states, so differences in transitions could be a function of any such differences rather than their baseline smoking and vaping status. We were also unable to take into account potential confounders such as tobacco/nicotine dependence, previous quit attempts, reasons for vaping, or motivation to quit smoking, mainly owing to small sample sizes in some groups. Finally, this paper is only a descriptive examination of smokers/vapers, therefore a causal interpretation of patterns is premature. Forthcoming papers will analyze the interplay between cigarettes and NVPs over time using methods that have greater potential for directly addressing possible explanations for the patterns of transitions presented in this initial descriptive study.

\section{Conclusions}

Longitudinal cohort studies are essential for assessing transitions in tobacco/nicotine product use over time, and for assessing the potential of NVPs and other nicotine delivery products for reducing 
the harms of smoking. Our international study confirms that the vast majority of smokers were still smoking 18 months later despite the availability of less harmful alternatives, demonstrating the persistence of cigarette smoking, the most dangerous and commonly used nicotine product. This study has also highlighted several differences between daily and non-daily smokers, particularly that non-daily smokers were more likely than daily smokers to have discontinued smoking. Notably, among daily smokers, vaping did not improve rates of discontinued smoking, but it appears to have been helpful in reducing daily smoking to non-daily smoking. Relapse rates were low among the ex-smokers in this study, and there were no differences in relapse between those who were vaping at baseline compared to those who were not vaping. Further longitudinal research is needed to examine the utility of vaping as an aid to quit smoking and if vaping can be helpful in relapse prevention. Moreover, considering the low rates of discontinued smoking in all four countries, reinforcing the need for continued public health focus on cigarette smoking prevention and cessation efforts is imperative.

Author Contributions: Conceptualization: S.G., G.T.F., K.M.C., A.H., G.M., R.B.; Methodology: M.E.T., C.B., G.T.F., J.O., N.M., S.G., A.C.K.Q.; Formal analysis: G.M., S.G., G.T.F.; Investigation: G.M., S.G, G.T.F., K.M.C., R.B., A.H., K.A.K.; Writing-original draft preparation: S.G., G.T.F.; Writing-review and editing: S.G., G.M., K.M.C., A.H., R.B., D.H., R.J.O., M.L.G., K.A.K., A.M., M.E.T., S.C.H., D.T.L., J.F.T., A.C.K.Q., N.M., J.O., C.B., G.T.F.; Supervision: G.T.F; Project administration: A.C.K.Q., J.O., N.M.; Funding acquisition: K.M.C., G.T.F., R.J.O., R.B., D.H., D.T.L., M.E.T.; All authors have approved the published version of the manuscript.

Funding: This study was supported by grants from the US National Cancer Institute (P01 CA200512), the Canadian Institutes of Health Research (FDN-148477), and the National Health and Medical Research Council of Australia (APP 1106451). GTF was supported by a Senior Investigator Award from the Ontario Institute for Cancer Research (IA-004). RO and AH are supported by a Tobacco Centers of Regulatory Science US National Cancer Institute grant (U54 CA238110).

Acknowledgments: The authors would like to acknowledge and thank all those that contributed to the International Tobacco Control Four Country Smoking and Vaping Survey (ITC 4CV) Survey: all study investigators and collaborators, and the project staff at their respective institutions. The authors would also like to sincerely extend their appreciation to the reviewers for the time spent, and their insightful feedback, on this paper.

Conflicts of Interest: K.M.C. has received payment as a consultant to Pfizer, Inc., for service on an external advisory panel to assess ways to improve smoking cessation delivery in healthcare settings. K.M.C. also has served as paid expert witness in litigation filed against cigarette manufacturers. G.T.F., D.H., and J.F.T. have served as expert witnesses on behalf of governments in litigation involving the tobacco industry. M.L.G. has received a research grant from Pfizer and served as a member of scientific advisory board to Johnson and Johnson. A.M. is a UK National Institute for Health Research (NIHR) Senior Investigator. The views expressed in this article are those of the authors and not necessarily those of the NIHR, or the UK Department of Health and Social Care. All other authors have no conflicts of interest to declare.

\section{References}

1. U.S. Department of Health and Human Services. Smoking Cessation. A Report of the Surgeon General; U.S. Department of Health and Human Services, Centers for Disease Control and Prevention, National Center for Chronic Disease Prevention and Health Promotion, Office on Smoking and Health: Atlanta, GA, USA, 2020.

2. Hatsukami, D.K.; Carroll, D.M. Tobacco harm reduction: Past history, current controversies and a proposed approach for the future. Prev. Med. 2020, 106099. [CrossRef] [PubMed]

3. Foundation for a Smoke-Free World. Global Trends in Nicotine. 2018. Available online: https://www. smokefreeworld.org/advancing-industry-transformation/global-trends-nicotine/ (accessed on 7 July 2020).

4. McNeill, A.; Brose, L.S.; Calder, R.; Bauld, L.; Robson, D. Evidence Review of e-Cigarettes and Heated Tobacco Products 2018. A Report Commissioned by Public Health England; Public Health England: London, UK, 2018. Available online: https://assets.publishing.service.gov.uk/government/uploads/system/uploads/attachment_ data/file/684963/Evidence_review_of_e-cigarettes_and_heated_tobacco_products_2018.pdf (accessed on 7 July 2020).

5. The National Academies of Sciences, Engineering and Medicine (NASEM). Public Health Consequences of E-Cigarettes. 2018. Available online: https://www.nap.edu/catalog/24952/public-health-consequences-of-ecigarettes (accessed on 7 July 2020). 
6. Hajek, P.; Phillips-Waller, A.; Przulj, D.; Pesola, F.; Smith, K.M.; Bisal, N.; Li, J.; Parrott, S.; Sasieni, P.; Dawkins, L.; et al. A Randomized Trial of E-Cigarettes versus Nicotine-Replacement Therapy. N. Engl. J. Med. 2019, 380, 629-637. [CrossRef] [PubMed]

7. Walker, N.; Parag, V.; Verbiest, M.; Laking, G.; Laugesen, M.; Bullen, C. Nicotine patches used in combination with e-cigarettes (with and without nicotine) for smoking cessation: A pragmatic, randomised trial. Lancet Respir. Med. 2020, 8, 54-64. [CrossRef]

8. King, B.A. The chicken or the egg? The value of longitudinal research in an increasingly diverse tobacco product landscape. Tob. Control 2020, 29, S131-S133. [CrossRef] [PubMed]

9. Hyland, A.; Kasza, K.A.; Borek, N.; Kimmel, H.L.; A Taylor, K.; Compton, W.M.; Day, H.; A Donaldson, E.; Sharma, E.; Anic, G.; et al. Overview of tobacco use transitions for population health. Tob. Control 2020, 29, S134-S138. [CrossRef]

10. Taylor, K.A.; Sharma, E.; Edwards, K.C.; Halenar, M.J.; Kissin, W.; Kasza, K.A.; Day, H.; Anic, G.; Gardner, L.; Hammad, H.T.; et al. Longitudinal pathways of exclusive and polytobacco cigarette use among youth, young adults and adults in the USA: Findings from the PATH Study Waves 1-3 (2013-2016). Tob. Control 2020, 29, S139-S146. [CrossRef]

11. Kasza, K.A.; Borek, N.; Conway, K.P.; Goniewicz, M.L.; Stanton, C.A.; Sharma, E.; Fong, G.T.; Abrams, D.B.; Coleman, B.N.; Schneller, L.M.; et al. Transitions in Tobacco Product Use by U.S. Adults between 2013-2014 and 2014-2015: Findings from the PATH Study Wave 1 and Wave 2. Int. J. Environ. Res. Public Health 2018, 15, 2515. [CrossRef]

12. Coleman, B.N.; Rostron, B.; Johnson, S.E.; Persoskie, A.; Pearson, J.L.; Stanton, C.A.; Choi, K.; Anic, G.; Goniewicz, M.L.; Cummings, K.M.; et al. Transitions in electronic cigarette use among adults in the Population Assessment of Tobacco and Health (PATH) Study, Waves 1 and 2 (2013-2015). Tob. Control 2019, 28, 50-59. [CrossRef]

13. Kasza, K.A.; Edwards, K.C.; Tang, Z.; Stanton, C.A.; Sharma, E.; Halenar, M.J.; Taylor, K.A.; Donaldson, E.A.; Hull, L.C.; Bansal-Travers, M.; et al. Correlates of tobacco product cessation among youth and adults in the USA: Findings from the PATH Study Waves 1-3 (2013-2016). Tob. Control 2020, 29, S203-S215. [CrossRef]

14. McNeill, A.; Driezen, P.; Hitchman, S.C.; Cummings, K.M.; Fong, G.T.; Borland, R. Indicators of cigarette smoking dependence and relapse in former smokers who vape compared with those who do not: Findings from the 2016 International Tobacco Control Four Country Smoking and Vaping Survey. Addiction 2019, 114, 49-60. [CrossRef]

15. Strong, D.R.; Pearson, J.; Ehlke, S.; Kirchner, T.; Abrams, D.; Taylor, K.; Compton, W.M.; Conway, K.P.; Lambert, E.; Green, V.R.; et al. Indicators of dependence for different types of tobacco product users: Descriptive findings from Wave 1 (2013-2014) of the Population Assessment of Tobacco and Health (PATH) study. Drug Alcohol Depend. 2017, 178, 257-266. [CrossRef] [PubMed]

16. ITC Project. ITC Four Country Smoking and Vaping Survey Wave 1 (2016) Technical Report. University of Waterloo, Waterloo, ON, Canada; Medical University of South Carolina, Charleston, SC, USA; Cancer Council Victoria, Melbourne, Australia; King's College London, London, UK. 2017. Available online: https: //itcproject.s3.amazonaws.com/uploads/documents/4CV1_Technical_Report_Nov23201.pdf (accessed on 8 July 2020).

17. ITC Project. ITC Four Country Smoking and Vaping Survey, Wave 2 (2018) Technical Report. University of Waterloo, Waterloo, ON, Canada; Medical University of South Carolina, Charleston, South Carolina, USA; Cancer Council Victoria, Melbourne, Australia; the University of Queensland, Australia; King's College London, London, UK. Available online: https:/itcproject.org/methods/technical-reports/itc-four-countrysmoking-and-vaping-survey-wave-2-4cv2-technical-report/ (accessed on 8 July 2020).

18. Thompson, M.E.; Fong, G.T.; Boudreau, C.; Driezen, P.; Li, G.; Gravely, S.; Cummings, K.M.; Heckman, B.W.; O'Connor, R.; Thrasher, J.F.; et al. Methods of the ITC Four Country Smoking and Vaping Survey, Wave 1 (2016). Addiction 2019, 114, 6-14. [CrossRef] [PubMed]

19. Thompson, M.E. Using Longitudinal Complex Survey Data. Annu. Rev. Stat. Appl. 2015, 2, 305-320. [CrossRef]

20. Gravely, S.; Thrasher, J.F.; Cummings, K.M.; Ouimet, J.; McNeill, A.; Meng, G.; Lindblom, E.N.; Loewen, R.; O'Connor, R.J.; Thompson, M.E.; et al. Discussions between health professionals and smokers about nicotine vaping products: Results from the 2016 ITC Four Country Smoking and Vaping Survey. Addiction 2019, 114, 71-85. [CrossRef] [PubMed] 
21. UK Government. The Tobacco and Related Products Regulations 2016; UK Government: London, UK, 2016. Available online: https:/www.gov.uk/guidance/e-cigarettes-regulations-for-consumer-products (accessed on 1 September 2020).

22. Government of Canada. Tobacco and Vaping Products Act (TVPA). Available online: https://www.canada.ca/ en/health-canada/services/health-concerns/tobacco/legislation/federal-laws/tobacco-act.html (accessed on 7 July 2020).

23. Borland, R.; Murray, K.; Gravely, S.; Fong, G.T.; Thompson, M.E.; McNeill, A.; O'Connor, R.J.; Goniewicz, M.L.; Yong, H.-H.; Levy, D.T; et al. A new classification system for describing concurrent use of nicotine vaping products alongside cigarettes (so-called 'dual use'): Findings from the ITC-4 Country Smoking and Vaping wave 1 Survey. Addiction 2019, 114, 24-34. [CrossRef]

24. Kolenikov, S. Calibrating Survey Data using Iterative Proportional Fitting (Raking). Stata J. 2014, 14, 22-59. [CrossRef]

25. Muller, C.J.; MacLehose, R.F. Estimating predicted probabilities from logistic regression: Different methods correspond to different target populations. Int. J. Epidemiol. 2014, 43, 962-970. [CrossRef]

26. Columbia University Mailman School of Public Health. Population Health Methods. Difference-in-Difference Estimation. Available online: https:/www.publichealth.columbia.edu/research/ population-health-methods/difference-difference-estimation (accessed on 7 July 2020).

27. Swayampakala, K.; Thrasher, J.F.; Hardin, J.W.; Titus, A.R.; Liu, J.; Fong, G.T.; Fleischer, N.L. Factors associated with changing cigarette consumption patterns among low-intensity smokers: Longitudinal findings across four waves (2008-2012) of ITC Mexico Survey. Addict. Behav. Rep. 2018, 8, 154-163. [CrossRef]

28. Swayampakala, K.; Thrasher, J.; Carpenter, M.J.; Shigematsu, L.M.; Cupertio, A.P.; Berg, C.J. Level of cigarette consumption and quit behavior in a population of low-intensity smokers-longitudinal results from the International Tobacco Control (ITC) survey in Mexico. Addict. Behav. 2013, 38, 1958-1965. [CrossRef]

29. Hassmiller, K.M.; Warner, K.E.; Mendez, D.; Levy, D.T.; Romano, E. Nondaily smokers: Who are they? Am. J. Public Health 2003, 93, 1321-1327. [CrossRef]

30. Reyes-Guzman, C.M.; Pfeiffer, R.M.; Lubin, J.; Freedman, N.D.; Cleary, S.D.; Levine, P.H.; Caporaso, N.E. Determinants of Light and Intermittent Smoking in the United States: Results from Three Pooled National Health Surveys. Cancer Epidemiol. Biomark. Prev. 2017, 26, 228-239. [CrossRef] [PubMed]

31. Yong, H.-H.; Borland, R.; Cummings, K.M.; Gravely, S.; Thrasher, J.F.; McNeill, A.; Hitchman, S.C.; Greenhalgh, E.; Thompson, M.E.; Fong, G.T. Reasons for regular vaping and for its discontinuation among smokers and recent ex-smokers: Findings from the 2016 ITC Four Country Smoking and Vaping Survey. Addiction 2019, 114, 35-48. [CrossRef] [PubMed]

32. Augustson, E.M.; Marcus, S.E. Use of the Current Population Survey to characterize subpopulations of continued smokers: A national perspective on the "hardcore" smoker phenomenon. Nicot. Tob. Res. 2004, 6, 621-629. [CrossRef] [PubMed]

33. Simonavicius, E.; McNeill, A.; Brose, L.S. Transitions in smoking and nicotine use from 2016 to 2017 among a UK cohort of adult smokers and ex-smokers. Drug Alcohol Rev. 2020. [CrossRef]

34. Brose, L.S.; Hitchman, S.C.; Brown, J.; West, R.; McNeill, A. Is the use of electronic cigarettes while smoking associated with smoking cessation attempts, cessation and reduced cigarette consumption? A survey with a 1-year follow-up. Addiction 2015, 110, 1160-1168. [CrossRef]

35. Miller, C.R.; Smith, D.M.; Goniewicz, M.L. Changes in Nicotine Product Use among Dual Users of Tobacco and Electronic Cigarettes: Findings from the Population Assessment of Tobacco and Health (PATH) Study, 2013-2015. Subst. Use Misuse 2020, 55, 909-913. [CrossRef]

36. Brose, L.S.; Bowen, J.; McNeill, A.; Partos, T.R. Associations between vaping and relapse to smoking: Preliminary findings from a longitudinal survey in the UK. Harm Reduct. J. 2019, 16, 76. [CrossRef]

37. Herd, N.; Borland, R.; Hyland, A. Predictors of smoking relapse by duration of abstinence: Findings from the International Tobacco Control (ITC) Four Country Survey. Addiction 2009, 104, 2088-2099. [CrossRef]

38. Yong, H.H.; Borland, R.; Cummings, K.M.; Partos, T. Do predictors of smoking relapse change as a function of duration of abstinence? Findings from the United States, Canada, United Kingdom and Australia. Addiction 2018, 113, 1295-1304. [CrossRef]

39. National Institute for Health and Care Excellence (NICE). Stop Smoking Interventions and Services. NICE Guideline [NG92]. Advice on E-Cigarettes. 2018. Available online: https://www.nice.org.uk/guidance/ ng92/chapter/Recommendations\#advice-on-ecigarettes (accessed on 7 July 2020). 
40. Public Health England. E-Cigarettes: A Developing Public Health Consensus. Joint Statement on E-Cigarettes by Public Health England and Other UK Public Health Organisations. 2016. Available online: https://assets.publishing.service.gov.uk/government/uploads/system/uploads/attachment_ data/file/534708/E-cigarettes_joint_consensus_statement_2016.pdf (accessed on 7 July 2020).

41. Gravely, S.; Driezen, P.; Ouimet, J.; Quah, A.C.K.; Cummings, K.M.; Thompson, M.E.; Boudreau, C.; Hammond, D.; McNeill, A.; Borland, R.; et al. Prevalence of awareness, ever-use and current use of nicotine vaping products (NVPs) among adult current smokers and ex-smokers in 14 countries with differing regulations on sales and marketing of NVPs: Cross-sectional findings from the ITC Project. Addiction 2019, 114, 1060-1073. [CrossRef]

(C) 2020 by the authors. Licensee MDPI, Basel, Switzerland. This article is an open access article distributed under the terms and conditions of the Creative Commons Attribution (CC BY) license (http://creativecommons.org/licenses/by/4.0/). 